\title{
Review
}

\section{Current Researches on the Methods of Diagnosing Sasang Constitution: An Overview}

\author{
Si-Woo Lee ${ }^{1, *}$, Eun-Su Jang ${ }^{1, *}$, Jeon Lee $^{2}$ and Jong Yeol Kim \\ ${ }^{1}$ Korea Institute of Oriental Medicine and ${ }^{2}$ Department of Oriental Biomedical Engineering, \\ Daegu Haany University
}

\begin{abstract}
Sasang constitution diagnosis has traditionally been conducted by a Sasang constitutional medicine (SCM) doctor who examines the external appearance, temperament and various symptoms of an individual and then collectively analyzes this information to determine their own constitutions. However, because this process is subjective and not quantitative, many researchers have been attempting to develop objective and reasonable methods of determining constitutions. In Korea, even though a wide range of research regarding SCM has been conducted, most of the work has not been revealed internationally. So in this review, the authors have searched the Journal of Sasang Constitutional Medicine, as well as other Korean domestic journal databases and Pubmed for research regarding modernized constitution diagnosis methods so to provide the understanding of current research state and outlook for future research.
\end{abstract}

Keywords: body shape analysis - facial analysis - modernized constitution diagnosis methods questionnaires - Sasang constitutional medicine

\section{Introduction}

According to Donguisusebowon (longevity and life preservation in oriental medicine), all people belong to one of the four constitutions based on the four emotional energies of sorrow, anger, gladness and enjoyment, which are derived from Confucianism. It is possible to classify an individual's constitution based on their external appearance, temperament and various symptoms (1).

External appearance includes the body shape, as well as facial configuration and skin tactile. Temperament includes personality and abilities corresponding with the four emotional energies. Symptoms can be physiological or pathological. Spontaneously, an individual's constitution is not a simple conception, but a systematic

*These authors contributed equally to this work.

For reprints and all correspondence: Jong Yeol Kim, OMD, PhD, Korea Institute of Oriental Medicine (KIOM), 461-24 Jeonmin-dong, Yuseong-gu, Daejeon 305-811, Republic of Korea. Tel: +82-42-8689489; Fax: +82-42-868-9480; E-mail: ssmed@kiom.re.kr classification with its own unique psychological and physical traits (2).

The Sasang constitutional medicine (SCM), dividing human beings into four different constitution groups, is similar to Ayurveda, the traditional constitutional medicine of India, in which the individual's constitution is divided into three groups, based on their external appearance, skin condition, walking and eating style and emotional traits (3). While Western medicine focuses on diseases, Ayurveda and SCM have put the priority on the human being itself (4). Comparing these two constitutional medicines within the framework of disease prevention and quality-of-life (QOL) evaluation, Taeeumin (TE type) was found to be similar to Kapha type, Soyangin (SY type) to Pitta type, and Soeumin (SE type) to Vata type (5). Similar to Ayurveda, SCM is also fully dependent on the interpretation and the opinion of a doctor. So, to overcome this drawback, many SCMrelated researchers have attempted to develop modernized diagnostic tools to support objective and quantitative constitution diagnosis. 
The Korea Institute of Oriental Medicine (KIOM) and other domestic oriental medical colleges have been continuously evaluating SCM for the past 10 years, and their efforts to develop objective constitution diagnosis methods have received much attention (6). Recently, due to advances in information and communication technologies, it has become possible to process large volumes of genetic data. However, the results of these researches have not been introduced to the international CAM academic community. Therefore, the authors conducted this review of current researches regarding constitution diagnosis methods to provide an understanding of SCM as well as to provide an outlook for the future of SCM.

\section{Methods}

Articles related to constitution diagnosis were searched with the keyword 'Sasang' in Pubmed and in the databases containing literature regarding oriental medicine written in Korean. In addition, all articles in the Journal of Sasang Constitutional Medicine (JSCM) were reviewed from the first issue in 1989 up to the latest article in 2008. And, by examining the abstract, methods and results of all articles found in keyword-matching search, specific researches, which cover the modernization of traditional constitution diagnosis methods based on clinical experiment, were strategized to be selected and evaluated.

In Donguisusebowon, Lee Je-ma suggested the following methods to determine patients' Sasang constitutions: physical appearance (體形氣像), features and way of speaking (容貌詞氣), temperament and talent (性質才幹) and pathological syndromes and pharmacology (病證藥理). So the selected ones were then reclassified into following four categories based on those methods: body shape analysis, facial analysis, voice analysis and questionnaire.

\section{Search Results}

(i) In JSCM, 86 articles were finally selected from 730 articles, based on the authors' analyses of the abstracts, methods and contents. These articles covered the modernization of traditional constitution diagnosis methods that evaluate the body shape, face, voice and questionnaire.

(ii) In Pubmed, 22 articles were found using 'Sasang' as the keyword. Out of the 22, one article that dealt with the development of constitution diagnosis method was selected for this review.

(iii) By another search in databases containing journals related to oriental medicine written in Korean, such as DBPIA (http://www.dbpia.co.kr), KISS (http:// kiss.kstudy.com) and KISTI (http://society.kisti. re.kr), 278 articles using 'Sasang' as a keyword
Table 1. Selected researches from various databases

\begin{tabular}{lccc}
\hline $\begin{array}{l}\text { Name of DB or } \\
\text { Journal searched }\end{array}$ & $\begin{array}{l}\text { Number of selected } \\
\text { articles for review }\end{array}$ & $\begin{array}{l}\text { Number of searched } \\
\text { SCM articles }\end{array}$ & Language \\
\hline JSCM & 86 & 730 & Korean \\
Pubmed & 1 & 22 & English \\
DBPIA & 6 & 69 & Korean \\
KISS & 5 & 72 & Korean \\
KISTI & 29 & 137 & Korean \\
Total & 127 & 1030 & \\
\hline
\end{tabular}

were found. Then, 40 articles, which dealt with the development of constitution diagnosis methods, were extracted for this review.

Final search results are summarized in Table 1.

\section{Results}

\section{Body Shape Analysis}

Lee Je-ma (1837-1900) proclaimed that human beings could be classified into four constitutions, Taeyangin (TY type), Tae-eumin (TE type), Soyangin (SY type) and Soeumin (SE type), based on functional activity differences between the four viscera-lungs, liver, spleen and kidneys, which induce different shapes at four parts of the body. As a result, Taeyangins have a more developed 'lung area' and a less developed 'liver area', while Taeeumins have a more developed 'liver area' and a less developed 'lung area'. In addition, Soyangins have a more developed 'spleen area' and a less developed 'kidney area', while Soeumins have a more developed 'kidney area' and a less developed 'spleen area' (1).

In body shape analysis for constitution diagnosis there are two primary methods used, body trunk measurement and body segment measurement (Table 2). These two methods deal with morphological differences in the trunk or body segments among constitutions.

As for body trunk measurement, Huh et al. (7) suggested that trunk shape differed among individuals with different Sasang constitutions; therefore, SCM doctors could classify Sasang constitutions based on the lengths of five horizontal lines in the trunk derived from Lee Je-ma's Four Area Theory. They reported that the accuracy of constitution diagnosis using this method was $\sim 90 \%$ when conducted by highly skilled practitioners (8). However, this method had a weak point that the measured lengths of anterior trunk are not sufficient for checking the degree of development in the trunk. Therefore, some researchers have insisted that the eight circumferences of the trunk embody the four viscera area better than the five lengths of the anterior trunk $(9,10)$.

However, manual measuring methods cause inconvenience to the patients, and various human errors like intra-observer and inter-observer discrepancy could 
Table 2. Body shape analysis researches

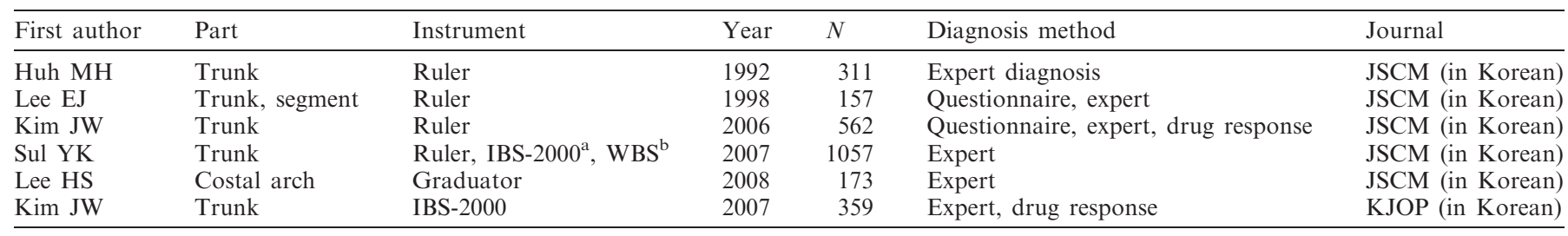

KJOP: Korean Journal of Oriental Physiology and Pathology; N: number of participants.

${ }^{\mathrm{a}}$ IBS-2000: 3D (Three-Dimensional) Body Scanner of the Way of Moire; ${ }^{\mathrm{b}}$ WBS - Whole Body Scanner System.

Table 3. Face analysis researches

\begin{tabular}{lllrrl}
\hline First author & Method & Instrument & Year & $N$ & Diagnosis method \\
\hline Koh BH & 2D & Camera (Nikon FM2) & 1996 & 170 & Questionnaire, herbal prescription, experts \\
Hong SC & 3D & Camera (Nikon FM2) with moire slit & 1998 & 113 & Questionnaire, herbal prescription, experts \\
Hong SC & 2D & Camera (Nikon FM2) & 1998 & 209 & Questionnaire, herbal prescription, experts \\
Hong SC & 3D & Camera (Nikon FM2) with moire slit & 1998 & 98 & Questionnaire, herbal prescription, experts \\
Yoon JH & 2D & Digital Camera (Kodak CD265) & 2000 & 846 & Questionnaire, herbal prescription, experts \\
Kwak CK & 3D & 3D-FARA & \\
Seok JH & 2D & Digital Camera (Nikon D200) & 2006 & - & - \\
Kwak CK & 3D & 3D-AFRA & 2007 & 1732 & Questionnaire, herbal prescription, experts \\
\hline
\end{tabular}

$N$ : number of participants.

aD-FARA: 3D Face Automatic Recognition Apparatus; 'bD-AFRA: 3D Automatic Face Recognition Apparatus.

influence the constitution diagnosis. Therefore, there has been a need to develop an automatic and reliable measuring method (11). Recently, an automatic measuring device, IBS 2000 (Z-Scan Co. Ltd, Pusan, Korea), was developed and was clinically evaluated. The results of several researches have shown that the differences between automatic measurement using the IBS 2000 and manual measurement by skilled operators are minimal $(11,12)$.

Other researchers tried to find various specific body segments correlated to constitutions such as head, neck, thigh, lower leg, foot and upper arm as well as the angle between both costal margins over the abdomen, for convenient clinical use $(13,14)$.

\section{Facial Analysis}

Since the Neijing era, oriental medical doctors have stressed diagnoses that involve observing the shape and color of the face. SCM doctors also have maintained this viewpoint and have elaborated facial analysis for determining the constitution or evaluating the patient's health. Meanwhile, the most challenging part about the descriptions in SCM literatures regarding whole facial appearance or the features of partial compartments of the face, such as ears, eyes, mouth and nose, is that they are subjective and non-quantitative (15). To overcome this handicap, many SCM doctors have made great efforts to make facial analysis more objective (Table 3 ).

The first quantitative attempt of constitution diagnosis based on facial analysis was made by Koh et al. in
1996. In their study, 200 indexes for classifying Sasang constitutions were suggested, which were generated with normalized lengths and their ratios of 69 feature points on the front and left lateral pictures of faces in the 178 participants (16). These 69 points had been established by SCM doctors through facial analysis based on Martin's facial points (17). Furthermore, some researchers have attempted to set up typical Korean faces to each Sasang constitution through analysis of the facial features of 846 participants $(18,19)$. However, facial-analysis methods using photographs are limited by lack of repeatability caused by the manual point assignment and discrepancy between a 2D image and SCM doctors' 3D recognition (15).

Therefore, to meet the needs for a realistic facial image, a stereo camera method was applied to facial analysis $(15,20)$. However, the results of several researches revealed that improvement was needed both in software and in hardware because it had a larger margin of error than 3D laser scan method $(19,21)$. So, instead of using the stereo camera method, 3D AFRA (3D automatic face recognition apparatus) that used a $3 \mathrm{D}$ shape restoration technique based on a space partition encoding optic formula was developed (22).

Recently, KIOM examined the correlation between constitutions and each possible distance, angle and distance ratio of the feature points in the frontal facial pictures. Of the 1225 distances, 40000 angles and 600000 distance ratios, 9 distances, 10 angles and 10 distance ratios were found to be statistically significant among constitutions. 


\section{Voice Analysis}

Oriental medical doctors generally have evaluated the patient's voice from the view of the 'five phases' to understand the characteristics of diseases (23). Indeed, the vocal tract characteristics are related to body shape, and the voice can differ according to the variations in the vocal tract. Lee Je-ma stressed careful observation of the patient's way of speaking as an important factor in constitutional classification. In addition, it is well-known that, due to state-of-the-art signal-processing techniques, it is possible to diagnose some diseases and identify an individual with a basic frequency and resonance components $(24,25)$.

In early research, Kim et al. recorded and analyzed the voice focusing on the pitch, several elements of basic frequency and talking speed (26). After this, many researches have been conducted to evaluate pitch, shimmer, harmonics, formant and energy of voice for constitution diagnosis, using CSL (computerized speech laboratory) and these researches have revealed that several factors differed significantly among constitutions (25-27). On the basis of these results, KIOM and Sangji University research teams have developed a voice analysis system, the PSSC-2004 (Phonetic System for Sasang Constitution-2004, VoiceOne Inc., Wonju, Korea). The PSSC-2004 can bring out the estimated membership degrees to each constitution by analyzing recorded voices.

The PSSC-2004 has been used to evaluate voice parameters such as pitch, APQ, octave, shimmer, energy, code and peak sum to determine constitutions $(28,29)$. Although PSSC-2004 was used as a Sasang constitution diagnosis method (25), its diagnosis accuracy is not sufficient for widespread use in clinical practice (Table 4).

\section{Questionnaires}

When SCM doctors diagnose a patient's constitution, it is very important to understand the patient's temperament. In the psychological field, questionnaires such as MBTI (Myers-Briggs Type Indicator) and TCI (Temperament and Character Inventory) have been widely used to analyze the patient's temperament. In the SCM field, there were also a lot of attempts to adopt questionnaire methods to Sasang constitution diagnosis (Table 5).
The first questionnaire developed for Sasang constitution diagnosis was the Constitution Diagnosis Questionnaire (I), which consists of various questions that have been commonly asked by SCM doctors in clinical practice (30). Following this, the QSCC (Questionnaire for Sasang Constitution Classification) was systematically developed by a group of experts comprised of oriental medical doctors and psychologists, which contained questions regarding the contents of Donguisusebowon. The answers provided by 241 constitution confirmed participants were then analyzed to develop optimal discriminant equations for constitution diagnosis (31). However, continued use of QSCC revealed that too many individuals were diagnosed as Taeyangins and its correspondence with the other (Sasang constitution diagnosis) questionnaires was quite low $(32,33)$.

To overcome the shortcomings of QSCC, the QSCC II was developed by oriental medical doctors, clinical psychologists, linguists and philosophers. They re-analyzed the contents of Donguisusebowon and Gyeokchigo (manuscript on science by Lee Je-ma), and then added questions to QSCC that had been proven to be statistically significant. The accuracy of QSCC II was initially reported to be $70.8 \%$ (31). However, subsequent researches revealed that QSCC II had a lower accuracy rate than claimed by the developers $(34,35)$. Furthermore, it was later revealed that QSCC II could not diagnose Taeyangins (36).

Some researchers have reported that many of the questions in QSCC II pertaining to temperament had minor roles, and those pertaining to external features such as body shape had decisive roles $(36,37)$. Therefore, there

Table 4. Voice analysis researches

\begin{tabular}{llrrll}
\hline First author & Instrument & Year & $N$ & Diagnosis method & Journal \\
\hline Yang SH & CSL $^{\mathrm{a}}$ & 1996 & 66 & Questionnaire & JSCM \\
Kim DL & CSL & 1998 & 134 & Questionnaire & JSCM \\
Shin MR & CSL & 1999 & 140 & Questionnaire, expert & JSCM \\
Yang SM & CSL & 2001 & 30 & Questionnaire & JSCM \\
Park SJ & CSL & 2004 & 71 & Questionnaire, expert & JSCM \\
Kim SH & PSSC-2004 & 2005 & 217 & Expert & JSCM \\
Kim DJ & PSSC-2004 & 2005 & 231 & Expert & JSCM \\
Bae HS & PSSC-2004 & 2007 & 529 & PSSC-2004 & JSCM \\
\hline
\end{tabular}

$N$ : number of participants.

${ }^{\mathrm{a} C S L}$ - Computerized Speech Lab Model 4300B (KAY USA); ${ }^{\text {b} P S S C-2004-P S S C-2004, ~ E M C-909 ~(J A P A N), ~ C o m p u t e r ~ w i t h ~}$ Samsung Sound Card.

Table 5. Questionnaire-related researches

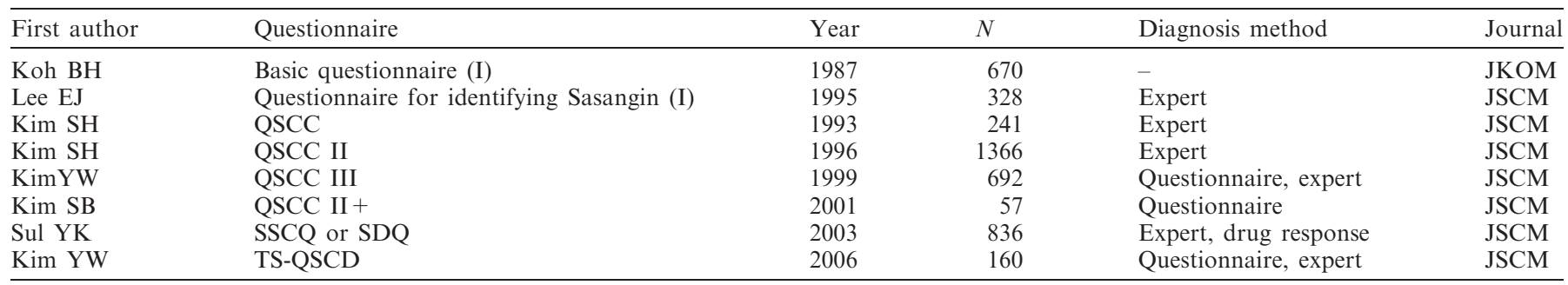

JKOM: Journal of Korean Oriental Medicine; $N$ : number of participants. 
has been increasing needs for developing a new questionnaire in which more questions associated with external features are included.

Recently, KIOM and the Korean Society for SCM have released a web-based diagnosis program based on the new questionnaire, SDQ (Sasangin Diagnosis Questionnaire). This questionnaire was divided into seperate sections for doctors and patients. Furthermore, the questionnaire contained few questions regarding temperament, but more quantitative items, such as the input of the actual measurements of the patient's body. As a result, SDQ overcame many of the problems associated with QSCC II. The reliability of SDQ has been established using statistical methods based on clinical data (38).

\section{Discussion}

One of the largest problems associated with CAM is the lack of objectivity in the diagnosis. SCM, which is similar to the other areas of CAM, has a subjective diagnosis process. Therefore, it is difficult for SCM to be accepted by mainstream medicine. To overcome this limitation in SCM, researchers have attempted to develop objective constitution diagnosis tools in many ways.

In the early 'body shape' and 'facial' analyses, researchers adapted manual measurement to their research, which has been recently applied with automatic measurement to reduce the margin of error in manual measurement. It is expected that a new product will be released within the next 2-3 years.

Regarding voice analysis, even with the advances in voice analysis technology, the vocal factors for constitution diagnosis are not yet clear. If the vocal factors that increase the accuracy in the diagnosis can be identified, it could be possible to easily combine it with other constitution diagnosis methods.

In the questionnaire research, clinical factors such as external features, temperament and symptoms have been mainly reflected in the questions. And in recent research, more quantitative and objective factors such as body shape and facial features have been included in the questionnaire, so that it evolves to the integrative constitutional diagnosis method that traditional SCM doctors have tried to pursue for a long time.

Individual diagnosis tools have shown the possibilities in diagnosing constitution to some degree, but the accuracy of each tool cannot reach the level that is sufficient for SCM doctors to utilize it in clinical practice. It is known that the statistical accuracy of individual diagnosis tools, is a little low compared to SCM experts' diagnosis, for example, $52 \%$ in body analysis (10), 58\% in voice analysis (39) and unknown in facial analysis.

This is because Sasang constitution diagnosis should be done through a combination of information about the body shape, face, voice, character and symptoms by SCM doctors as Lee Je-ma described in his book (1) (Fig. 1). Furthermore, SCM doctors sometimes need

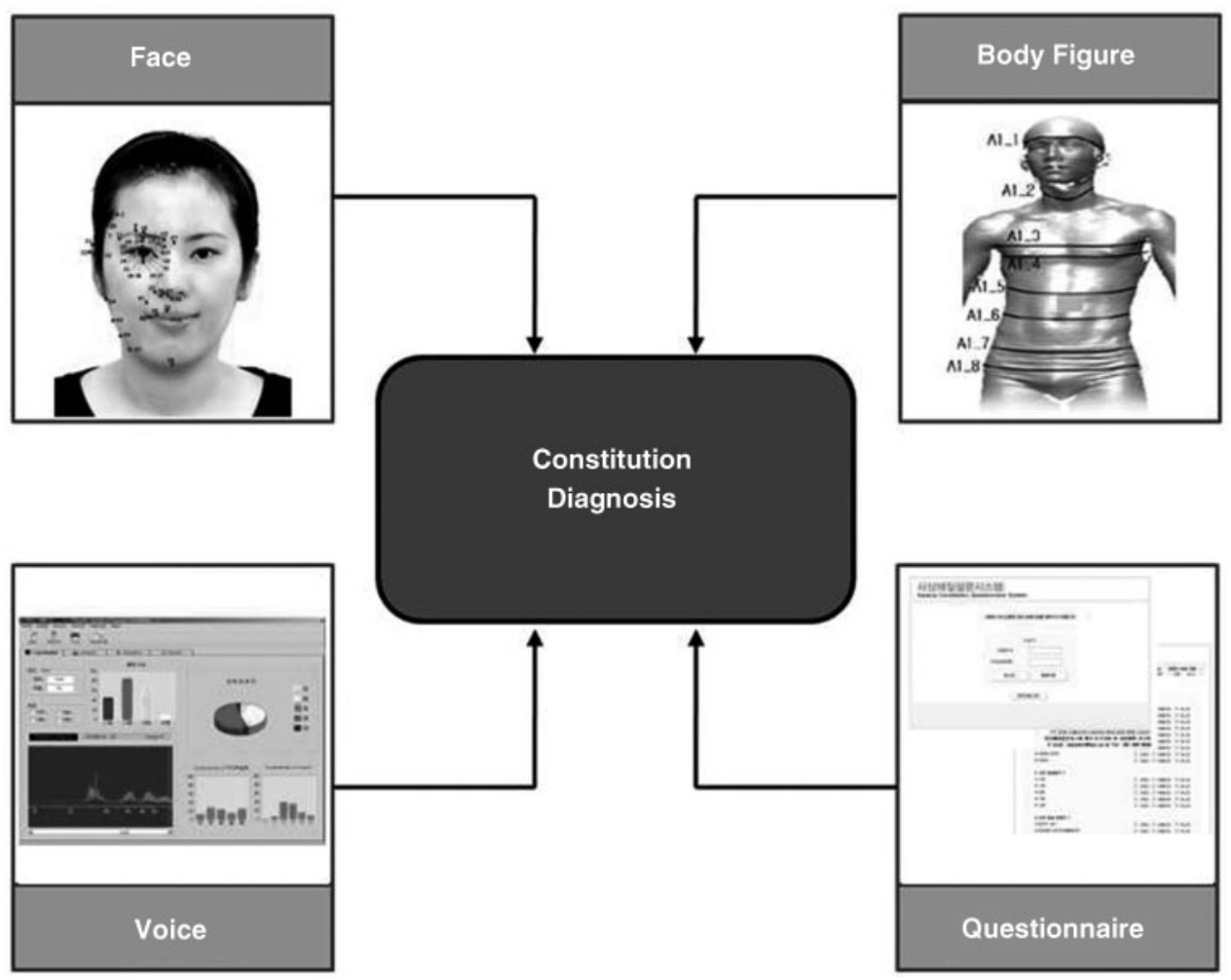

Figure 1. The future of Sasang constitution diagnosis combining of the body shape, face, voice and questionnaire. 
more information about the patients' response to constitutional herbal medication to confirm their diagnosis.

KIOM has collected the participants' data that includes a questionnaire, facial pictures, body shape measurements and voice recording with the standard operation procedures through multiple collaborating centers $(40,41)$. Using this information, KIOM constructed a database that is called Constitution Information Bank (CIB). KIOM will make a pilot integrated tool to diagnose Sasang constitutions in clinical practice by the end of 2009.

\section{Funding}

Korea Science and Engineering Foundation (KOSEF) grant funded by the Korean government (MEST) (Grant No. M10643020001-08N4302-00100).

\section{References}

1. Lee JM. In: Choi SH (tr). Longevity \& Life Preservation in Oriental Medicine. Seoul: Kyung Hee University Press, 1996.

2. Chae H, Lyoo IK, Lee SJ, Cho SH, Bae HS, Hong MC, et al. An alternative way to individualized medicine: psychological and physical traits of Sasang typology. J Altern Complement Med 2003;9:519-28.

3. Joshi RR. A biostatistical approach to ayurveda: quantifying the tridosha. J Altern Complement Med 2004;10:879-89.

4. Patwardhan B, Warude D, Pushpangadan P, Bhatt N. Ayurveda and traditional Chinese medicine: a comparative overview. eCAM 2005;2:465-73.

5. Kim DK. A comparative study of Korean oriental medicine \& Indian traditional medicine. J Korean Orient Med 2005;26:201-16 (in Korean)

6. Shim EB, Lee SW, Kim JY, Earm YE. Physiome and Sasang constitutional medicine. J Physiol Sci 2008;58:433-40.

7. Huh MH, Song JM, Kim DL, Koh BH. A study on the morphological schematization of Sasangin. J Sasang Constitut Med 1992;4:107-48 (in Korean).

8. Huh MH, Koh BH, Song IB. The body measuring method to classify Sasang constitutions. J Sasang Constitut Med 2002;14:5-66 (in Korean).

9. Kim JW, Yeo HR, Kim KK, Jeon SH, Lee MH, Lee YT. Study on the characteristics of body shapes classified by Sasang constitution according to age group. Korean $J$ Orient. Physiol Pathol 2007;21:258-62 (in Korean).

10. Kim JW, Jeon SH, Sul YK, Kim KK, Lee EJ. A study on the body shape classified by Sasang constitutions and gender using physical measurement. J Sasang Constitut Med 2006;18:54-61 (in Korean).

11. Sul YK, Lee EJ, Kim KK, Kim JW. Comparative study of Sasang constitutions diagnostic accuracy rate on measurement method of body shape by IBS-2000 compensator. J Sasang Constitut Med. 2007;19:78-89 (in Korean).

12. Kim JW, Sul YK, Choi JJ, Kwon SD, Kim KK, Lee YT. Comparative study of diagnostic accuracy rate by Sasang constitution on measurement method of body shape. Korean $J$ Orient Physiol Pathol 2007;21:338-56 (in Korean).

13. Lee EJ, Lee JK, Kim JY, Song JM. The study on the biomechanical body segmental parameters of Korean adults with reference to Sasang constitutional medicine. J Sasang Constitut Med 1998;10: 143-60 (in Korean).

14. Lee HS, Park SS. A morphological study of angle of costal arch according to the Sasang constitution. J Sasang Constitut Med 2008;20:48-55 (in Korean).
15. Lee EJ, Park YB, Kwak CK, Yoo JH, Kim JW, Kim KK, et al. The study of face model and type. J Sasang Constitut Med 2006;18:25-33 (in Korean).

16. Koh BH, Song IB, Chou YJ, Choi CS, Kim JW, Hong SC, et al. The morphological characteristics of head and face according to Sasang constitution. J Sasang Constitut Med 1996;8:101-86 (in Korean).

17. Martin R. Lerbuch der Anthropologie, Zeitewr Band: Kraniologie, Osteologie. Jena, Germany: Verlag Gustav Fischer, 1928 (in German)

18. Yoon JH, Lee SK, Lee EJ, Koh BH, Song IB. Morphological standardization research of head and face on the 50's and 60's in Korean according to Sasang constitution. J Sasang Constitut Med 2000;12:123-31 (in Korean).

19. Seok JH, Song JH, Kim HJ, Yoo JH, Kwak CK, Lee JH, et al. An hardware error analysis of 3D automatic face recognition apparatus (3D-AFRA): surface reconstruction. J Sasang Constitut Med 2007:19:30-9 (in Korean)

20. Kwak CK, Cho YB, Sohn EH, Yoo JH, Koh BH, Kim JW, et al. An positioning error analysis of $3 \mathrm{D}$ face recognition apparatus. $J$ Sasang Constitut Med 2006;18:34-40 (in Korean).

21. Kwak CK, Cho YB, Sohn EH, Yoo JH, Koh BH, Kim JW, et al. An hardware error analysis of 3D face automatic recognition apparatus (3D-AFRA). J Sasang Constitut Med 2006;18:49-56 (in Korean).

22. Kwak CK, Seok JH, Song JH, Kim HJ, Hwang MW, Yoo JH, et al. An error analysis of the 3D automatic face recognition apparatus (3D-AFRA) hardware. J Sasang Constitut Med 2007;19:22-9 (in Korean).

23. Yang YK. Huangdi Neijing Suwen. Seoul, Korea: Seongbosa, 1980, 35-52 (in Chinese).

24. Smith A. Whisperings of ovarian cancer: acknowledging women's voices. Clin J Oncol Nurs 2008;12:913-20.

25. Shin MR, Kim DL. An study on the correlation between sound characteristics and Sasang constitution by CSL (Computerized Speech Lab). J Sasang Constitut Med 1999;11:137-57 (in Korean).

26. Yang SH, Kim DL. A study on the correlation between sound characteristic and Sasang constitution. J Sasang Constitut Med 1996;8:191-202 (in Korean).

27. Kim D, Park SS, Kwon GR. An objective study of Sasang constitution diagnosis by sound analysis. J Sasang Constitut Med 1998;10:65-80 (in Korean)

28. Kim DJ, Jung WK, Choi JW, Kim DL, Jeon JW. A study on the characteristics of the adult men sound as by Sasang constitution analyzed with PSSC-2004. J Sasang Constitut Med 2005;17:67-83 (in Korean).

29. Kim SH, Han DY, Youn JY, Kim DL, Jeon JW. A Study on the characteristics of Korea adult women sound as by Sasang constitution analysed with PSSC-2004. J Sasang Constitut Med 2005;17: 84-102 (in Korean).

30. Koh BH, Song IB. A study on the method of Sasang constitution classification. J Korean Orient Med 1985;8:146-50 (in Korean).

31. Kim SH, Kho BH, Song IB. A study on the validation of questionnaire for Sasang constitution classification (QSCC). J Sasang Constitut Med 1993;5:67-85 (in Korean).

32. Lee SK, Lee EJ, Hong SC, Koh BH. An analysis on the characteristics of Sasang constitution. J Sasang Constitut Med 1996;8:349-76 (in Korean).

33. Park SH, Park EK, Choi JY. Analysis of inter-questionaire agreement in determining Sasang constitution. J Sasang Constitut Med 1999;11:103-17 (in Korean).

34. Kim SB, Lee SK, Lee EJ, Koh BH, Song IL. A study on the validity to make a diagnosis of soeumin by QSCC II. J Sasang Constitut Med 2000;12:94-103 (in Korean).

35. Park HS, Ju JC, Kim JH, Kim KY. A study on clinical application of QSCC II. J Sasang Constitut Med 2002;14:35-44 (in Korean).

36. Park EK, Park SS. A study on comparison of responses to the questionnaire based on Sasang institution's differences questionnaire of Sasang constitution classification(I). J Sasang Constitut Med 2000;12:157-72 (in Korean). 
37. Kim TY, Yoo JH, Lee EJ, Koh BH, Song IB. The study on the upgrade of QSCC II(1). J Sasang Constitut Med 2003;15:27-38 (in Korean).

38. Yoo JH, Kim JW, Kim KK, Kim JY, Koh BH, Lee EJ. Sasangin diagnosis questionnaire: test of reliability. J Altern Complement Med 2007;13:111-22 (in Korean).

39. Jang ES, Baek YH, Kim HS, Lee SW. The study on sensitivity and specificity of Sasang constitution diagnosis methods. Korean $J$ Orient Med 2007;19:125-31 (in Korean).
40. Kang JH, Yoo JH, Kim JY. A study on the correlation between body-size and MDVP parameters in the normal male and female Korean population. $J$ Voice Sci 2008;15:107-19 (in Korean).

41. Koo IH, Kim JY, Kim MG, Kim KH. Feature selection from a facial image for distinction of Sasang constitution. eCAM 2009 (KIOM Suppl).

Received March 13, 2009; accepted June 23, 2009 


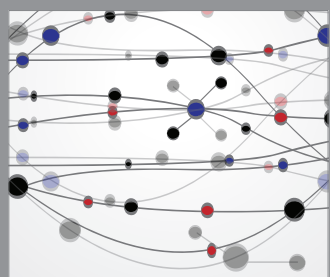

The Scientific World Journal
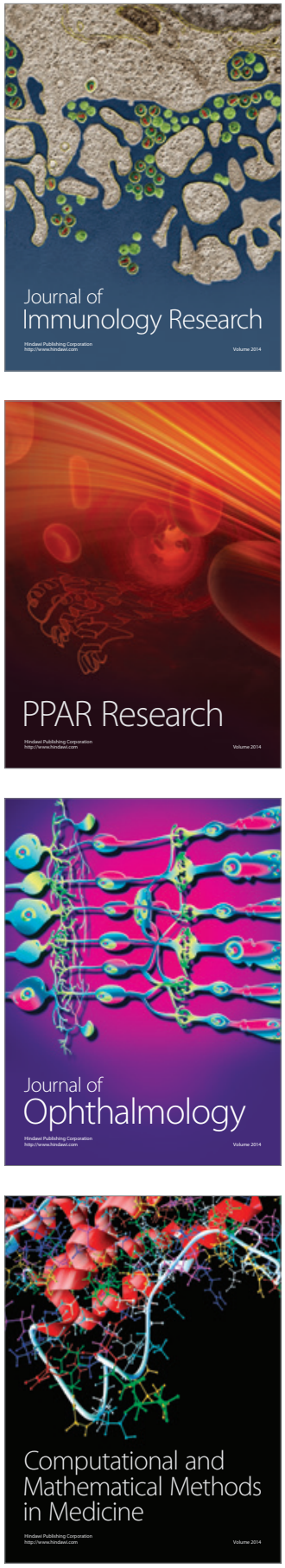

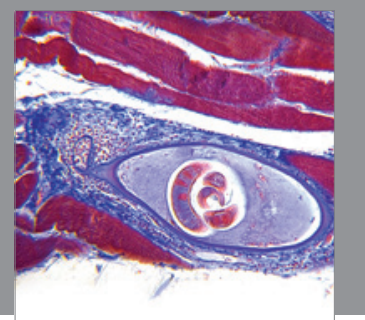

Gastroenterology

Research and Practice
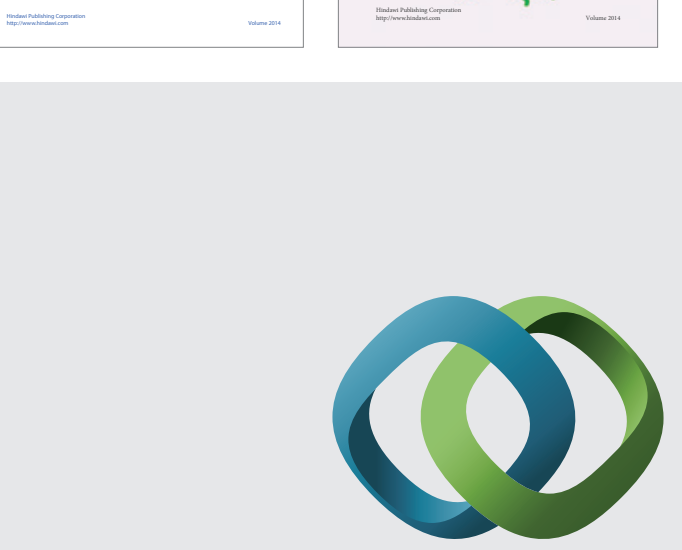

\section{Hindawi}

Submit your manuscripts at

http://www.hindawi.com
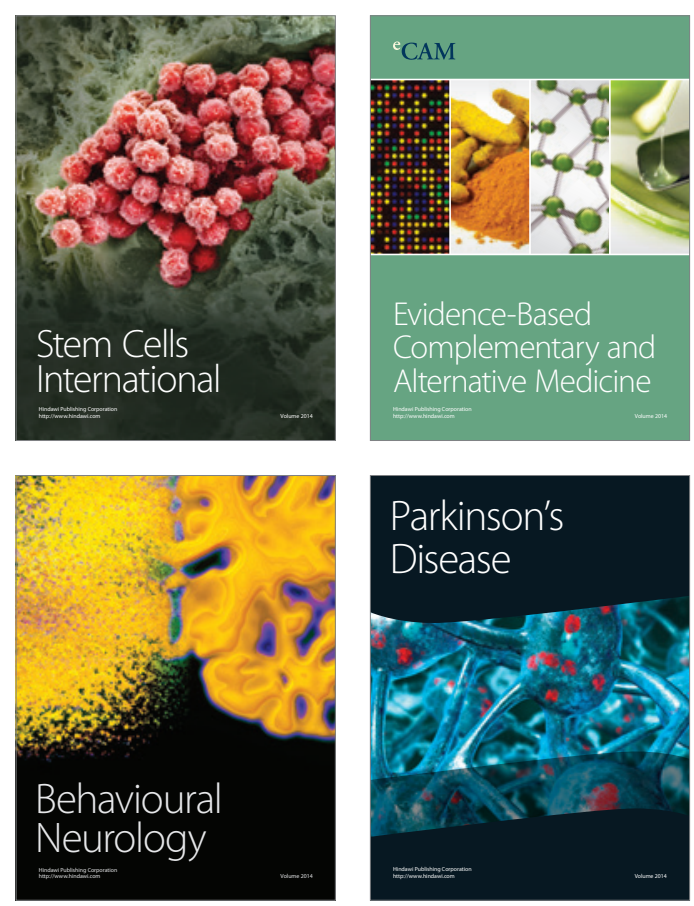

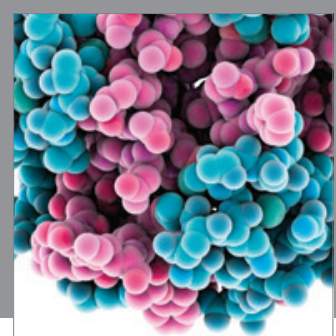

Journal of
Diabetes Research

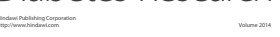

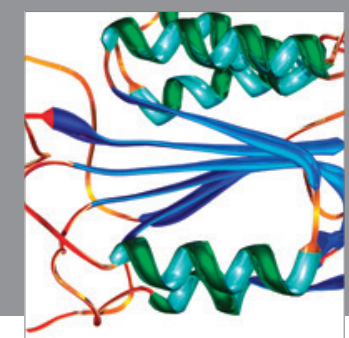

Disease Markers
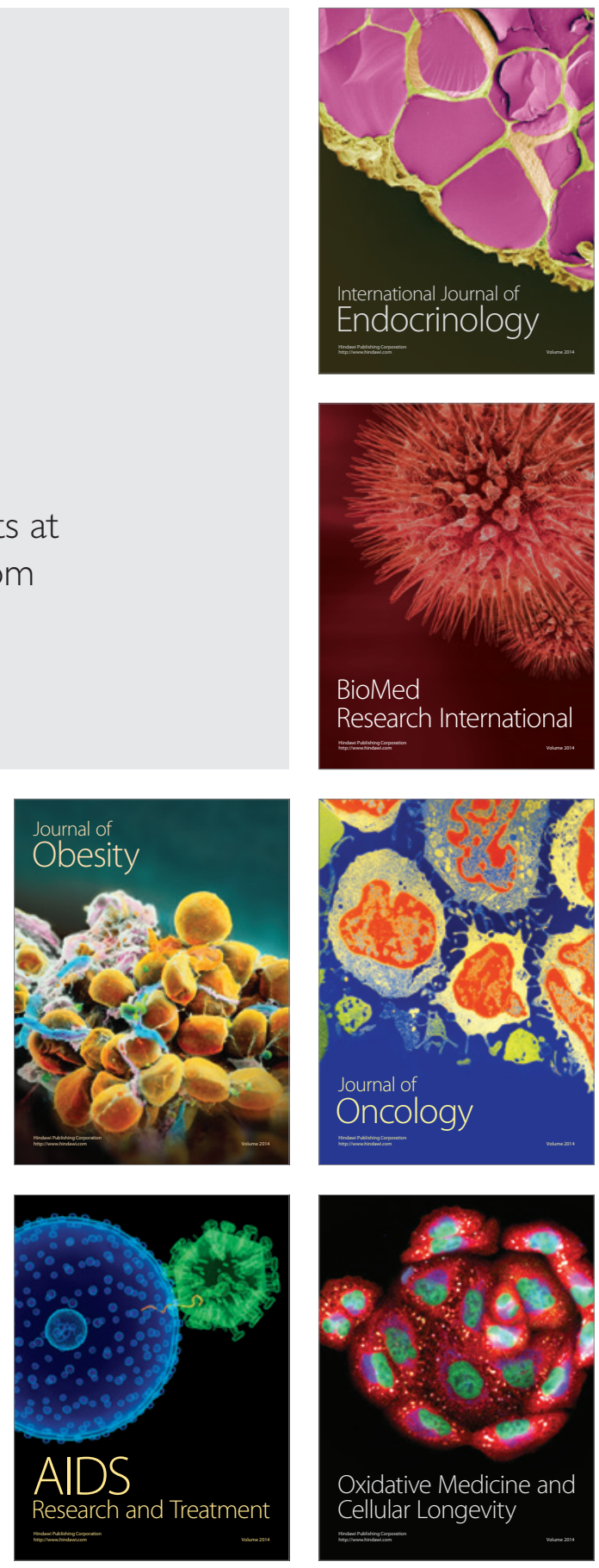